\title{
Erratum
}

\section{Free Actions of Finite Groups on Varieties. II}

William Browder and Nicholas M. Katz

Math. Ann. 260, 403-412 (1982)

In our example 3.3(1), line 6, it is asserted, falsely, that $\operatorname{deg}(X)=N^{g}$. In fact, $\operatorname{deg}(X)=(g !) N^{g}$, and it is rather the coherent Euler characteristic $\chi\left(X, \mathscr{L}^{\otimes N}\right)$ which is $N^{g}$. The two sentences following this error become correct if "deg $(X)$ " be replaced by " $\chi\left(X, \mathscr{L}^{\otimes N}\right)$ "; this results from the following theorem, which is proven but not stated in our paper.

Theorem. Let $k$ be an algebraically closed field, $X$ a projective $k$-scheme with $H^{0}\left(X, \mathcal{O}_{X}\right)=k$, and $G$ a finite group of $k$-automorphisms of $X$ which acts freely on $X$. For any invertible sheaf $\mathscr{L}$ on $X$ whose isomorphism class in $\operatorname{Pic}(X)$ is fixed by $G$, we have

1) $\# G$ divides $\chi(X, \mathscr{L})^{2}$.

2) if $G$ is cyclic, or if $\operatorname{char}(k)=p>0$ and $G$ is a p-group, then $\# G$ divides $\chi(X, \mathscr{L})$.

The point of example $3.3(1)$ is that this theorem is sharp for principally polarized abelian varieties. 